\title{
(INVITED) mm-Wave Silicon ICs: an Opportunity for Holistic Design
}

\author{
Ali Hajimiri \\ California Institute of Technology, Pasadena, CA 91125, USA
}

\begin{abstract}
Millimeter-waves integrated circuits offer a unique opportunity for a holistic design approach encompassing RF, ana$\log$, and digital, as well as radiation and electromagnetics. The ability to deal with the complete system from the digital circuitry to on-chip antennas and everything in between offers unparalleled opportunities for completely new architectures and topologies, previously impossible due the traditional partitioning of various blocks in conventional design. This opens a plethora of new architectural and system level innovation within the integrated circuit platform. This paper reviews some of the challenges and opportunities for mm-wave ICs and presents several solutions to them.
\end{abstract}

\section{INTRODUCTION}

We have witnessed a phenomenal growth since the initial introduction of integrated circuit [1]-[4]. New advancements in silicon integrated circuits has made it possible to integrate billions of nano-scale transistors capable of operating at mm-wave frequencies on a single die, thus creating an unprecedented opportunity to devise systems that leverage a very large number of transistors at mm-waves. Although conventional system architectures and their associated partitioning (e.g., antenna, filter, front-end, synthesizer, and digital back-end) can be directly ported to an integrated setting, the true potential of such integrated platforms can only be fully realized through a holistic co-design at multiple levels of abstraction (e.g., EM, RF, and digital) that could result in superior overall system architectures.

There are many challenges to full integration of mm-wave systems in silicon, despite the obvious and numerous advantages of such integration [6]. Conventional silicon integrated circuit processes have a conductive substrate, which can cause energy loss due to magnetically induced eddy currents. Antennas can also couple energy into the substrate resulting in some additional loss. Furthermore, the finite conductivity of the metal structures used in passive devices results in additional energy loss in the system. The conductors' ohmic loss can be significantly more important at mm-waves because of the small skin depth (e.g., the skin depth of copper at $60 \mathrm{GHz}$ is approximately $300 \mathrm{~nm}$ ). This is particularly problematic in CMP processes which results in rougher surfaces. The additional "fill" and "cheese" rules for wide metal strips also degrade the current handling capabilities of the metal lines.

The other equally important effect is the high dielectric constant of most semiconductor substrates, such as silicon $\left(\varepsilon_{r}=11.7\right)$. The rectangular silicon substrate forms a dielectric waveguide that can sustain propagating modes depending on its physical dimensions. Unfortunately, many of the natural modes for a typical silicon die size fall right in the middle of the mm-wave bands of interest. This creates an alternative mechanism for energy loss for on-chip components, where energy could easily leak into the substrate modes and be dissipated by ohmic loss within the substrate, or even worse, be radiated in undesirable directions [5][6][8]. Also, energy can easily be coupled back into other on-chip components creating potentially strong parasitic coupling between seemingly unrelated elements on the same die. Special care must be paid to the overall design to avoid such coupling, by using techniques such as fully-differential signalling to minimize generation and pick-up of mm-wave signals.
For an on-chip antenna, the high dielectric constant of the substrate causes a considerable portion of the electromagnetic energy to be sucked into the substrate as opposed to being radiated into the air from the top side [8]. In the case of silicon, the impact of this effect is further exacerbated by the conductivity of the substrate. This is a very important issue when trying to integrate the antennas with the rest of the chip.

Power generation in small feature size silicon technologies also presents a very serious challenge, mostly due to the low transistor breakdown voltages resulting from the scaling process and the shrinking of the depletion regions in the transistors. This necessitates the use of a lower supply voltage. Unfortunately, this is in direct conflict with the maximum power that can be generated using conventional power amplifier techniques, necessitating the use of parallel structures and novel power combing approaches, such as those described in [9][10].

Obviously, the modeling of the transistors (as well as the passive devices) becomes more challenging at these higher frequencies $[11][12][13]$. The smaller parasitic components within the models are more prone to error, and hence special attention must be paid to guarantee reliable results, especially in the presence of process variations and environmental changes.

Even in the absence of the aforementioned challenges of making mm-wave integrated circuits in silicon, we still need to be concerned about the mm-wave interface to the outside world. External connections to the chip need to be rethought completely. As an example, consider the case where we connect the mm-wave input (or output) of the chip to a perfectly matched transmission line on a adjacent printed circuit board using a wirebond. Approximating a $1 \mathrm{~mm}$ long wirebond as a $1 \mathrm{nH}$ inductor with a $Q$ of 30, we find that our connection has an impedance of approximately $12 \Omega+j 360 \Omega$ at $60 \mathrm{GHz}$, making it very difficult to achieve a proper impedance match without techniques similar to those in [14]. Furthermore, such an element can easily turn into an unintended radiative element at $\mathrm{mm}$-wave frequencies, creating very serious problems at the system level. Even a solder ball in flipchip technology presents a non-trivial impedance to match to the outside world. Reported results based on direct wafer probing of the die entirely ignore this challenge. If one tries to make the interface with wirebond, every effort should be made to make it into a structure emulating a transmission line, as in [14]. On the other hand, these interface challenges make the totally-integrated solutions with on-chip antenna much more attractive by offering a solution where the high frequency electrical interface to the outside world is completely eliminated and replaced by an electromagnetic radiating interface. An example of such an approach can be found in [8][15].

All of the above considerations demonstrate the important challenges in the area of mm-wave integrated circuits. It is clear that the direct application of traditional MMIC approaches to design of silicon-based integrated circuits, is neither efficient, nor optimum, since such techniques are fundamentally based on the underlying assumption of using a small number of well-characterized transistors. At the same time, direct application of lower frequency analog techniques to this problem would not be particularly successful either, as in the absence of proper treatment of 
the complex electromagnetics issues involved in such systems, the designers will not be able to devise implementations that closely match the expected results. Thus, it is clear that to take full advantage of this significant opportunity, one needs to start at the system architecture level and adopt new holistic methodologies and architectures along the way. We will discuss two examples of such a holistic approach in the rest of this paper.

\section{INTEGRATED PHASED ARRAYS}

Integration of a complete phased array system in silicon results in substantial improvements in cost, size, and reliability. It also offers various opportunities to perform on-chip signal processing and conditioning without having to go off-chip, leading to additional savings in cost and power. The multiple signal paths, operating in harmony, provide benefits at the system and circuit level.

Multiple antenna phased-arrays imitate a directional antenna whose bearing can be controlled electronically [16]-[21]. This electronic steering makes it possible to emulate antenna properties such as gain and directionality, while eliminating the need for continuous mechanical reorientation of the actual antennas. Additionally, the parallel nature of a phased array antenna transceiver alleviates the power handling and noise requirements for individual active devices used in the array. In the past, such systems have been implemented using a large number of microwave modules, adding to their cost and manufacturing complexity [19][20].

A phased-array transmitter or receiver consists of several signal paths each connected to a separate antenna. The antenna elements of the array can be arranged in different spatial configurations [18]. The array can be formed in one, two, or even three dimensions, with one or a two dimensional array being more common.

For narrowband systems, the time delay necessary in each element of a phased-array can be approximated by a phase-shift. This approximation leads to some signal dispersion, due to the non-constant group delay, which increases as the bandwidth of the signal increases. This dispersion translates to a higher BER in communication systems and lower resolution in radar systems [22].

The phase shift necessary in each element of a phased-array can be achieved at RF, at baseband/IF or in the LO path. In integrated implementations, there are several advantages to using LO path phase shifting, as the gain in each element of the transmitter or receiver is less sensitive to the amplitude variations at the LO ports of the mixers [14][22].

The various phases of the LO necessary in the LO-phase shifting approach can be generated in a central location by generating all the necessary phases at one place (e.g, a multi-phase oscillator), as in [14][22], or in a decentralized fashion by distributing only one phase of the LO signal and generating the remaining phases locally using a phase rotator for each LO path, similar to [8][15].

A fully integrated 4-element phased array transceiver with onchip antennas has been designed and fabricated in a $130 \mathrm{~nm} \mathrm{SiGe}$ BiCMOS process [8][15]. The receiver consists of the complete down-conversion path with low-noise amplifier (LNA), frequency synthesizer, phase rotators, combining amplifiers, and onchip dipole antennas. A distributed active combining amplifier at an IF of $26 \mathrm{GHz}$ is used to perform the signal combining. A 52$\mathrm{GHz}$ first LO is generated on chip and is routed to different elements, where it is phase shifted locally by the phase rotators. The local LO-path phase-shifting scheme enables a robust distribution network that scales well with increasing frequency and number of elements while providing high-resolution phase shifts. Measurements indicate a single-element LNA gain of $23 \mathrm{~dB}$ and a noise

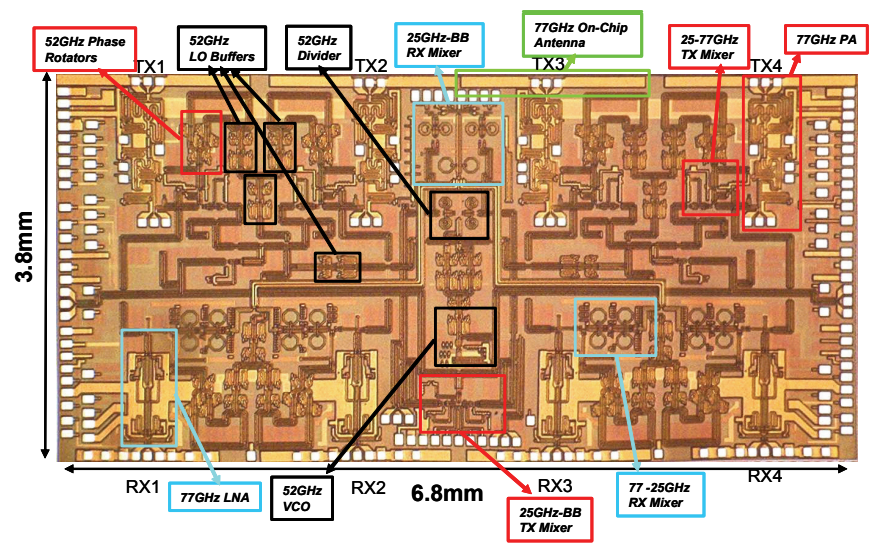

Fig. 1 Die photo of the phased-array transceiver with on-chip antennas

figure of $6.0 \mathrm{~dB}$. Each of the four receive paths has a gain of 37 $\mathrm{dB}$ and a single-path overall noise figure of $8.0 \mathrm{~dB}$. Each on-chip antenna has a gain of $+8 \mathrm{dBi}$ [23]. Each element of the 2-step upconversion transmitter generates $+12.5 \mathrm{dBm}$ of output power at $77 \mathrm{GHz}$ with a bandwidth of $2.5 \mathrm{GHz}$ leading to a 4-element effective isotropic radiated power (EIRP) of $+24.5 \mathrm{dBm}$. Each on-chip PA has a maximum saturated power of $+17.5 \mathrm{dBm}$ at 77 $\mathrm{GHz}$. The entire phased array transceiver occupies an area of $3.8 \mathrm{~mm} \times 6.8 \mathrm{~mm}$, as shown in the die photo of Fig. 1. This fully integrated phased array system demonstrates the potentials of silicon integration at mm-wave frequencies. The next example shows another example of the holistic design approach.

\section{NEAR-FIELD DIGITAL ANTENNA MODULATION}

Conventional radio transmitters modulate the desired information at the baseband and then up-convert and amplify that information by modulating the desired signal to create the desired phase and amplitude for the symbols transmitted. This requires upconversion mixers and perhaps more importantly a linear (or linearized) PA for non-constant-envelope modulations. The modulated signal is fed to an antenna that radiates the modulated signal in all directions, albeit with different gain factors and time delays. Even in the case of a directional antenna, although a stronger signal is sent in the desired direction, essentially the same data constellation and information is sent in other directions that can be intercepted by an unintended receiver with good sensitivity.

The near-field digital antenna modulation technique is a fundamentally different approach to modulating and transmitting data that combines digital circuitry and electromagnetics to modulate the signal directly at the antenna through digital manipulation of the electromagnetic boundary conditions [24]. The digital antenna modulation makes it possible to simplify the rest of the transmitter by eliminating the need for upconversion mixers and linear power amplifiers. It can be used to create a secure communication channel by sending the desired data only in the intended direction, while a scrambled data stream is transmitted in other directions. Also, it can be used to concurrently transmit two (or multiple) streams of completely independent data in different directions both at the maximum symbol rate, increasing the transmitted data rate significantly. As we will see next, its very simple, yet elegant, architecture allows it to be used for transmitting data at very high rates not limited by the bandwidth of the upconversion chain.

Fig. 2 shows the basis principle behind the digital antenna modulation technique, where an a dipole antenna is driven by a $\mathrm{CW}$ signal of constant amplitude and phase. There is also a conduc- 


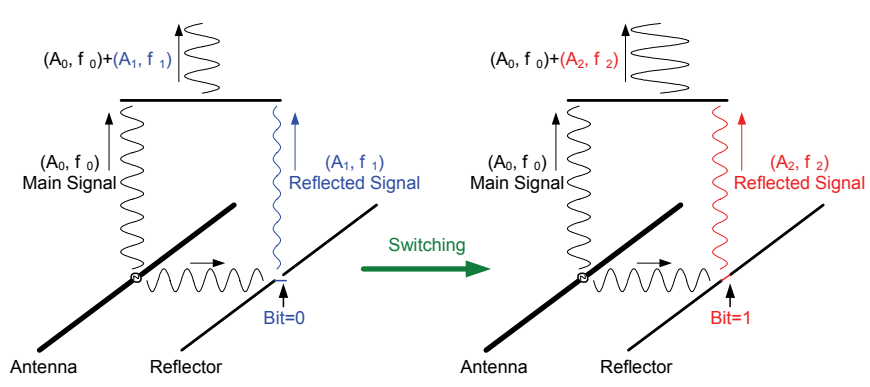

Fig. 2 Single bit modulation of the transmitted signal by switching the reflector in the near field.

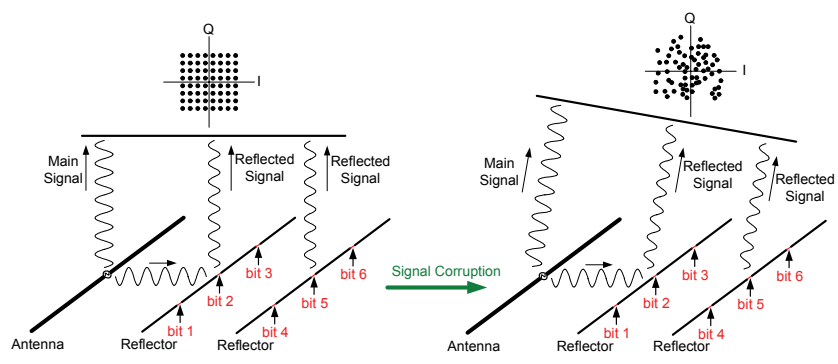

Fig. 3 Multiple reflectors with multiple switches to create different constellation points in a) the intended direction and b) in the unintended one [24].

tive metal line with comparable dimensions to the wavelength (a reflector) next to the antenna which can be shorted or opened at some point along its length using a switch. The reflected signal interferes with the main signal radiated by the antenna in a given direction. The amplitude and phase of the reflected signal depend on the boundary conditions that the reflector imposes and can be varied by turning the digital switch on or off. The two states of the switch result in two different phases and amplitudes in the direction of interest hence generating two distinct points in the IQ plane. This provides a crude but simple one-bit digital modulation without changing the output power or phase of the PA driving the antenna hence allowing it to operate at its highest efficiency.

Although the single-reflector single-switch configuration of Fig. 2 provides a basic binary modulation, it offers limited control over where the two modulation points fall. More control over the constellation point (i.e., transmitted phase and amplitude) can be obtained by introducing multiple reflectors each with multiple switches, as shown in Fig. 3. A large enough number of reflectors and switches in close proximity of the antenna makes it possible to create more constellation points and eventually achieve full coverage across the constellation.

It is noteworthy that a set of switch combinations that generates constellation points of the given modulation in the intended direction generates different constellation points in a different direction, as shown in Fig. 3b. This can be used to scramble the signal further in the unintended directions to implement a secure communication link by preventing an undesired eavesdropper from demodulating and recovering the signal.

For a large number of switches and reflectors, there are numerous different switch combinations that generate practically the same constellation point in a given direction. However, these different switch combinations can be used to generate distinct points in other directions simultaneously, allowing concurrent transmis-

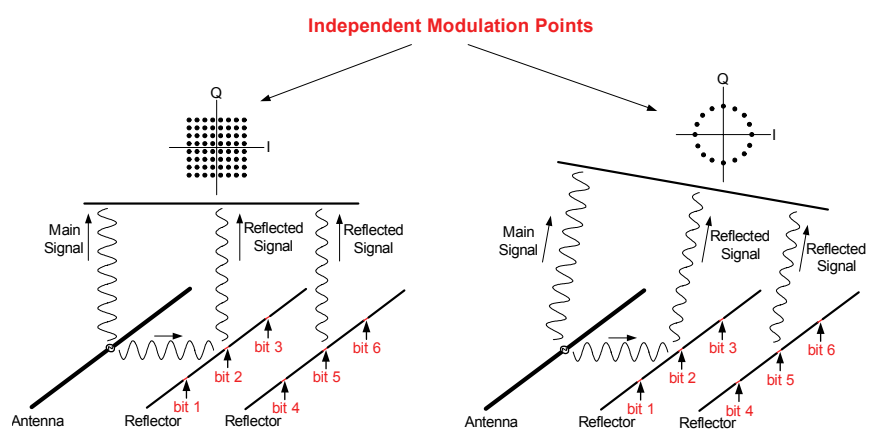

Fig. 4 Multiple reflectors with multiple switches to create different constellation points intended for two different receivers at two different angles.

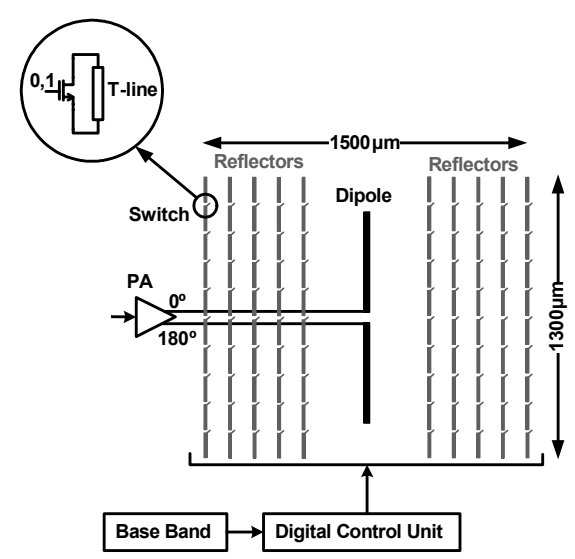

Fig. 5 The practical implementation of the digital antenna modulation scheme [24].

sion of two independent streams of data at full rate in two different directions, as depicted in Fig. 4.

The viability of this technique is demonstrated via an integrated circuit implementation [24] with an on-chip dipole antenna with 10 reflectors ( 5 on each side), each with 9 tuned MOS switches along its length, resulting in a total number of 90 switches and a extremely large $2^{90}\left(\sim 10^{27}\right)$ switching combinations, as illustrated in Fig. 5. The very large number of switching combinations provides numerous ways to generate a desired point on the constellation (phase and amplitude) in a given direction, providing many additional degrees of freedom that can be used for concurrency or security. The ability to simultaneously send independent information to several directions at full rate using a single transmitter is not achievable using the conventional transmitter architectures.

Making a high quality and fast integrated switch for use in mmwave frequencies is challenging. Achieving a small on-impedance requires a relatively large transistor size, which itself limits the maximum achievable off-impedance due to its large parasitic capacitance. This can be alleviated by resonating the transistor parasitic capacitance using a transmission line connected between its drain and its source (Fig. 5).

A transmitter at $60 \mathrm{GHz}$ using the direct antenna modulation scheme with an on-chip dipole antenna and reflectors is implemented in silicon. The antenna, the ten reflectors, the ninety switches, and the digital control circuitry for the switches occupy a die area of roughly $1.5 \mathrm{~mm} \times 1.5 \mathrm{~mm}$. The power is radiated 


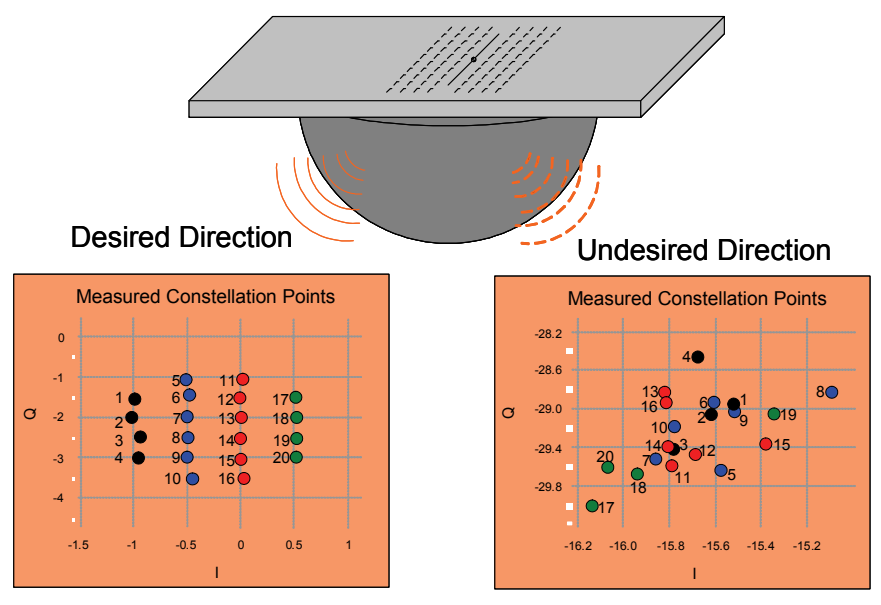

Fig. 6 The direct antenna modulation chip with a dielectric lens; a sample of the measured constellation points in the intended and unintended directions.

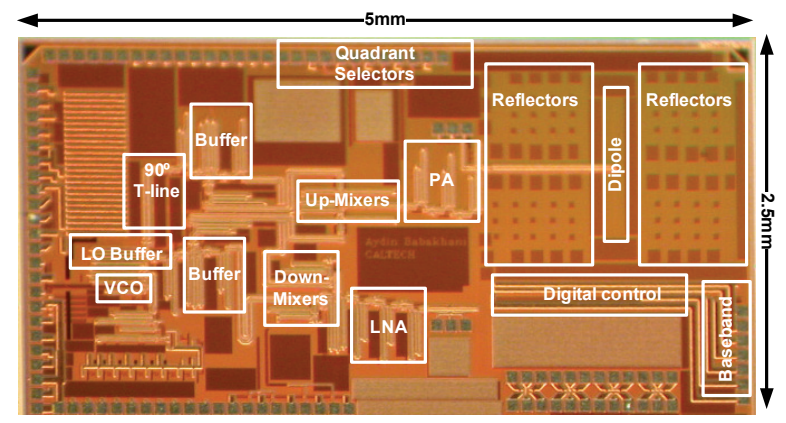

Fig. 7 The die micrograph of the direct antenna modulation transmitter.

from the backside of the chip with the aid of a hemispherical dielectric lens [5][8], as shown in Fig. 6.

An experimental demonstration of this technique is also shown in Fig. 6, where we show 20 points generated purely by switching the reflectors using 20 different switch combinations on the left hand side of Fig. 6. It should be noted that the input power and phase of the signal driving the antenna has not been changed to achieve the modulation. In addition, on the right side of Fig. 6, the received signal constellation at a different angle (about $90^{\circ}$ off) for the same set of switch combinations is plotted. It is obvious that these points are completely scrambled, making it practically impossible for a receiver at the undesirable angle to recover this signal, no matter how sensitive it may be. This is in contrast with a traditional transmitter that sends essentially the same modulated signal, albeit with different amplitude scales and phase epochs, in all directions. This measurement demonstrates the feasibility of the idea for security of communication links. The antenna is driven by a three stage fully-differential PA with an output power of at least $+7 \mathrm{dBm}$. The die photo is shown in Fig. 7 .

It is self-evident at this point that the ability to switch the antenna reflectors in the near field using a large number of very fast switches is a direct result of our ability to integrate, antennas, RF, and digital circuits on the same substrate and breaking of the conventionsal partitioning of the system. This is an example of the holistic co-design of electromagnetic structures with analog and digital circuitry. In practice, designing such system pose chal- lenges not only at the architecture and circuit levels, but also with respect to the simulation tools and methodology.

\section{CONCLUSIONS}

For the first time, it is possible to have a practically unlimited number of extremely fast and reliable transistors capable of operating at mm-wave frequencies in close proximity of structures comparable to the wavelengths of interest on the same silicon substrate, where electromagnetics, analog, and digital circuits can be intertwined deeply. This unique combination makes is possible to consider novel holistic approaches to communication and radar systems operating at mm-wave frequencies only limited by our imagination and creativity.

\section{ACKNOWLEDGMENTS}

The author thanks the contributions of A. Babakhani, Y. Wang, and H. Wang, Prof. D. B. Rutledge, Prof. S. Weinreb of Caltech and Dr. A. Natarajan, Dr. I. Aoki, Dr. S. Kee, Dr. A. Komijani, Dr. X. Guan, Prof. H. Hashemi, Prof. J. Buckwalter, and Prof. E. Afshari formerly of Caltech for the numerous contribution to Caltech's mm-wave activities. We have benefitted from the support of Caltech's Lee Center for Advance Networking, National Science Foundation, and DARPA Trusted Foundry Program.

\section{REFERENCES}

[1] J. S. Kilby, United States Patent Number 3,138,743, Filed Feb. 6, 1959, Granted June 23, 1964.

[2] J. S. Kilby, United States Patent Number 3,261,081, Filed Feb. 6, 1959, Granted July 19,1966

[3] R. F. Stewart, United States Patent Number 3,138,747, Filed Feb. 12, 1959 , Granted June 23, 1964

[4] G.E. Moore, "Cramming more components onto integrated circuits," Electronics, vol. 38, No. 8, pp. 114-117, April 1965 .

[5] D. B. Rutledge, et al., "Integrated-Circuit Antennas," Infrared and MillimeterWaves, New York: Academic, pp. 1-90, 1983.

[6] A. Hajimiri, "mm-Wave Silicon ICs: Challenges and Opportunities," IEEE Custom Integrated Circuits Conference, Sept. 2007.

[7] A. Hajimiri, "Holistic Design in mm-Wave Silicon ICs," to appear in IEICE Transactions, 2008

[8] A Babakhani, X Guan, A Komijani, A Natarajan, and A. Hajimiri, "A 77-GHz Phased-Array Transceiver with On-Chip antennas in silicon: Receiver and Antennas," IEEE J. Solid-State Circuits, vol. 41, no. 12, pp. 2795-2806, Dec. 2006.

[9] I. Aoki, S.D. Kee, D.B. Rutledge, and A. Hajimiri, "Fully integrated CMOS powe amplifier design using the distributed active-transformer architecture," IEEE $J$ Solid-State Circuits, vol. 37, no. 3, pp. 371-383, March 2002.

[10] E. Afshari, H. Bhat, X. Li, A. Hajimiri, "Electrical funnel: A broadband signal combining method," Dig. International Solid-State circuits Conference, Feb. 2006

[11] X. Guan and A. Hajimiri, "A 24-GHz CMOS front-end," IEEE J. Solid-State Circuits, vol. 39, no. 2, pp. 368-373, Feb. 2003

[12] B. Floyd, s. Reynolds, U. Pfeiffer, T. Zwick, T. Beukema, and B. Gaucher, "SiGe Bipolar Transceiver Circuits Operating at 60GHz," IEEE J. Solid-State Circuits, vol. 40 , no. 1 , pp. $156-167$, Jan. 2005 .

[13] C. Doan, S. Emami, A. Niknejad, and R. Brodersen, "Millimeter Wave CMOS Design," IEEE J. Solid-State Circuits, vol. 40, no. 1, pp. 144-155, Jan. 2005

[14] H. Hashemi, X. Guan, A. Komijani, and A. Hajimiri, "A 24-GHz SiGe phasedarray receiver - LO phase shifting approach," IEEE Trans. Microwave Theory Tech, vol. 53, Issue 2, pp. 614-626, Feb. 2005.

[15] A Natarajan, A Komijani, X Guan, A Babakhani, and A. Hajimiri, "A 77-GHz Phased-Array Transceiver with On-Chip antennas in silicon: Transmitter and Local LO-Path Phase Shifting," IEEE J. Solid-State Circuits, vol. 41, no. 12, pp. 2807-2819, Dec. 2006.

[16] V. Aulock, W. H., "Properties of phased arrays," Proc. IRE, vol. 48, No. 10, pp. 1715-1728, Oct. 1960

[17] R. C. Hansen, Ed., Significant Phased Array Papers. Norwood, MA: Artech House, 1973

[18] R. S. Elliott, Antenna Theory and Design. Englewood Cliffs, NJ: Prentice-Hall, 1981.

[19] D. Parker, D. C. Zimmermann, "Phased arrays - part I: theory and architectures," IEEE Trans. Microwave Theory Tech, vol. 50, No. 3, pp 678-687, Mar. 2002.

[20] D. Parker, D. C. Zimmermann, "Phased-arrays - part II: implementations, applications, and future trends," IEEE Trans. Microwave Theory Tech., vol. 50, No. 3, pp 688-698, Mar. 2002.

[21] B. Kane, L. Geis, M. Wyatt, D. Copeland, and J. Mogensen, "Smart Phased Array SoCs: A Novel Application for Advanced SiGe HBT BiCMOS Technology," Proc. of IEEE, Vol. 93, no. 9, pp. 1656-1668, Sept. 2005.

[22] A. Hajimiri, A. Komijani, A. Natarajan, X. Guan, H. Hashemi, "Phased array systems in silicon," IEEE Comm. Mag., vol. 42, pp. 122-130, Aug. 2004

[23] A. Babakhani, D. B. Rutledge, and A. Hajimiri, "mm-Wave Phased Arrays in Silicon with Integrated Antennas," IEEE Proc. Ant. and Prop. Int. Symp., pp. 43694372, June 2007.

[24] A. Babakhani, D. B. Rutledge, and A. Hajimiri, "A Near-Field Modulation Technique Using Antenna Reflector Switching," ISSCC Digest of Technical Papers, pp. 188-189, Feb. 2008 